\title{
Probabilistic mapping reveals optimal stimulation site in essential tremor
}

Running head: Multicenter essential tremor stimulation map

Andreas Nowacki ${ }^{1 *}$, Sabry Barlatey ${ }^{1 \#}$, Bassam Al-Fatly², Till Dembek ${ }^{3}$, Maarten Bot ${ }^{4}$, Alexander L. Green MD ${ }^{5}$, Dorothee Kübler ${ }^{2}$, M. Lenard Lachenmayer ${ }^{6}$, Ines Debove ${ }^{6}$, Alba Segura-Amil ${ }^{1,7}$, Andreas Horn ${ }^{2}$, Veerle Visser-Vandewalle ${ }^{8}$, Rick Schuurman ${ }^{4}$, Michael Barbe $^{3}$, Tipu Z. Aziz ${ }^{5}$, Andrea A. Kühn², T.A. Khoa Nguyen ${ }^{1,7 \#}$, Claudio Pollo"\#

\# These authors contributed equally to this work

\section{Author affiliations:}

${ }^{1}$ Department of Neurosurgery, Inselspital, Bern University Hospital, University Bern, Bern, Switzerland

${ }^{2}$ Charite-Universitätsmedizin Berlin, Movement Disorders and Neuromodulation Unit, Department of Neurology, Germany

${ }^{3}$ Department of Neurology, University Hospital Cologne, Cologne, Germany

${ }^{4}$ Department of Neurosurgery, Academic Medical Center, Amsterdam, The Netherlands

${ }^{5}$ Nuffield Department of Clinical Neuroscience and Nuffield Department of Surgical Sciences, University of Oxford, Oxford, United King

${ }^{6}$ Department of Neurology, Inselspital, Bern University Hospital, University Bern, Bern, Switzerland

${ }^{7}$ ARTORG Center for Biomedical Engineering Research, University of Bern, Bern, Switzerland

8 Department of Stereotactic and Functional Neurosurgery, University Hospital Cologne, and University of Cologne, Faculty of Medicine, Cologne, Germany

\section{Corresponding author:}

Andreas Nowacki,

${ }^{*}$ Department of Neurosurgery, Inselspital, Bern University Hospital Bern, Switzerland

Telephone: (+)41316640230

e-mail address: neuro.nowacki@gmail.com

\section{Number of characters title: $\mathbf{7 4}$}

This article has been accepted for publication and undergone full peer review but has not been through the copyediting, typesetting, pagination and proofreading process which may lead to differences between this version and the Version of Record. Please cite this article as doi: $10.1002 /$ ana.26324 


\begin{abstract}
Objective: To obtain individual clinical and neuroimaging data of patients undergoing Deep Brain Stimulation for essential tremor from five different European centers to identify predictors of outcome and to identify an optimal stimulation site.
\end{abstract}

Methods: We analysed retrospectively baseline covariates, pre- and postoperative clinical tremor scores (12-month) as well as individual imaging data from 119 patients to obtain individual electrode positions and stimulation volumes. Individual imaging and clinical data was used to calculate a probabilistic stimulation map in normalized space using voxel-wise statistical analysis. Finally, we used this map to train a classifier to predict tremor improvement.

Results: Probabilistic mapping of stimulation effects yielded a statistically significant cluster that was associated with a tremor improvement greater than $50 \%$. This cluster of optimal stimulation extended from the posterior subthalamic area to the ventralis intermedius nucleus and coincided with a normative structural-connectivity-based cerebello-thalamic tract (CTT). The combined features "distance between the stimulation volume and the significant cluster" and "CTT activation" were used as a predictor of tremor improvement. This correctly classified a greater than $50 \%$ tremor improvement with a sensitivity of $89 \%$ and a specificity of $57 \%$.

Interpretation: Our multicentre ET probabilistic stimulation map identified an area of optimal stimulation along the course of the CTT. The results of this study are mainly descriptive until confirmed in independent datasets, ideally through prospective testing. This target will be made openly available and may be used to guide surgical planning and for computer-assisted programming of deep brain stimulation in the future. 


\section{Abbreviations:}

Anterior commissure $=\mathrm{AC}$

cerebello-thalamic tract $=$ CTT

Deep Brain Stimulation $=$ DBS

essential tremor $=\mathrm{ET}$

posterior commissure $=\mathrm{PC}$

midcommissural point $=\mathrm{MCP}$

posterior subthalamic area $=\mathrm{PSA}$

region of interest $=\mathrm{ROI}$

Subthalamic Nucleus $=$ STN

Tremor Rating Scale $=$ TRS

Volume of Tissue Activation $=$ VTA

Ventralis intermedius nucleus $=$ Vim

Ventralis oralis posterior nucleus $=$ Vop 


\section{Introduction}

Deep Brain Stimulation (DBS) is an established therapy for medication-refractory Essential Tremor syndrome (ET). The classical target is the Ventral intermediate nucleus (Vim) of the thalamus ${ }^{1}$. Early after the first published case series of thalamic DBS for ET in 1992, alternative targets inferior to the thalamus have been suggested by various groups including the caudal zona incerta, the prelemniscal radiations or - more generally - the posterior subthalamic area $(P S A)^{2-4}$. More recently, anatomical and neuroimaging studies based on diffusionweighted imaging and tractography have suggested that the cerebello-thalamic tract (CTT) might embody a common neuroanatomical substrate of stimulation-induced tremor alleviation ${ }^{5-}$ 7. These findings could implicate that instead of a confined anatomical (sub)area, stimulation at any point along the CTT-network will elicit optimal tremor-suppression. Depending on the intended target site of stimulation, reported follow-up and outcome measure, thalamic and subthalamic DBS have been reported to achieve an on average 48-73.8\% tremor reduction, ${ }^{9}$. Apart from the observed variability of outcomes between studies, there is also a considerable variability across studies about suboptimal and non-responders that have ranked up to $22 \%$. Furthermore, inconsistent and partly contradictory results were published regarding predictors of outcomes such as the preoperative tremor severity, age and stereotactic coordinates of the stimulating electrode ${ }^{8-10}$.

Hence, after almost 30 years of DBS for ET, it remains unclear if there are solid predictors of outcome and if there is an optimal stimulation site. Previous studies were mostly based on smaller and monocentric series of patients with presumably lower variability in electrode locations. However, a certain degree of variability in electrode placement and measured outcomes is a prerequisite for studies that aim to investigate relationships between stimulation sites and clinical outcomes. To this end, analysis of aggregated data from multiple centers with differing electrode targeting approaches would be appropriate. In this study, we gathered clinical and neuroimaging data of a large cohort of patients that underwent DBS for ET in five different centers to identify robust predictors of outcome and to identify an optimal stimulation site by applying probabilistic mapping. 


\section{Materials and methods}

\section{Patients}

This study retrospectively enrolled datasets of 119 patients with ET syndrome treated chronically with unilateral or bilateral DBS and operated at five different European DBS centres. Diagnosis of ET was assessed by specialized movement disorder neurologists according to recommended guidelines and indication for surgery was discussed at multidisciplinary boards ${ }^{11}$. Large parts of the data were recently published (13 patients from a randomized controlled, cross-over trial ${ }^{12}, 81$ from four retrospective studies ${ }^{13-16}$ ). Unpublished data from 25 additional patients were included where full datasets were available. Individual datasets were included in the present study if they contained: (1) preoperative tremor scores of validated clinical rating scales, (2) available baseline covariates (sex, age, disease duration), (3) 12 months postoperative tremor scores with stimulation on, (4) pre- and postoperative neuroimaging allowing reconstruction of lead location or individual AC-PC-coordinates of the reconstructed stimulating electrode, (4) stimulation parameters at 12 months following surgery. Exclusion criteria were a diagnosis of other tremor syndromes (Holmes tremor, dystonic tremor, Parkinson tremor) or a MRI-verified lesion in the cerebellum, thalamus or brain-stem. The study was conducted in accordance with the Declaration of Helsinki and approved by the institutional review board of the University Bern (KEK 2018-00841).

\section{Surgical Procedure and Clinical Evaluation}

In all patients electrodes (model 3389 or 3387, Medtronic Inc.; Boston Cartesia Vercise directional leads, Boston Scientific, or St. Jude 6148, St. Jude Medical) were implanted stereotactically into the Vim or PSA. The neurostimulation parameters were programmed according to best clinical practice by the local DBS neurologist. Preoperative "medication-off" and postoperative "stimulation-on, medication-off" tremor severities were assessed "nonblinded" based on the Fahn-Tolosa-Marin Tremor Rating Scale (TRS) in four centers and based on the Bain's tremor rating scale in one center by a specialized movement disorder neurologists at 12 months postoperatively ${ }^{17,18}$.

\section{Study outcomes}

The primary outcome measure was the percentage reduction of the Part A subscore for upper and lower extremities of the TRS or Bain's tremor rating scale per hemibody at 12 months following DBS lead implantation. 


\section{Lead Reconstruction and estimation of stimulation volumes}

DBS electrodes were reconstructed using the Lead-DBS toolbox (version 2.3.2) in Matlab 2019b (The Mathworks, Natick, MA, USA) ${ }^{19}$. Postoperative CT or MR images were linearly coregistered to the preoperative MRI using advanced normalization tools (ANT) with brain shift correction as implemented in Lead-DBS. Multispectral normalization to ICBM 2009b MNI space was carried out by applying the ANTs SyN Diffeomorphic Mapping ${ }^{20}$. This method was shown to segment the STN region with high precision comparable to manual expert segmentations in a recent comparative study ${ }^{21}$. DBS electrodes were automatically prereconstructed using the phantom-validated and fully-automated PaCER method ${ }^{22}$. All reconstructed electrodes were individually checked visually for plausibility by examining the postoperative electrode position on the original postoperative image in relation to reliably detectable landmarks by a neurosurgical fellow (SB) and a senior functional neurosurgeon (AN). In a minor number of cases (11 patients, 19 electrodes), Lead DBS failed to detect electrodes automatically or provided an aberrant reconstruction far outside the thalamosubthalamic region. In these specific cases, we compared the reconstruction result with the electrode position from the postoperative MRI or CT scan and manually corrected the electrode position according to the artifact based on the postoperative images in Lead DBS. For segmented electrodes, the orientation was determined with the Directional Orientation Detection (DiODe) algorithm that was validated in phantom models and clinical studies before $^{23,24}$. All VTAs were estimated with the SimBio/Fieldtrip pipeline directly in template space with the DISTAL atlas ${ }^{25}$. We applied the same VTA model and settings for all leads to facilitate computation. Default values were used for white matter and grey matter conductivities, 0.14 and $0.33 \mathrm{~S} / \mathrm{m}$, respectively, and a threshold of $0.2 \mathrm{~V} / \mathrm{mm}$.

\section{Stimulation map construction}

We computed a probabilistic stimulation map by aggregating individual stimulation volumes with their associated tremor improvement of the contralateral hemibody at 12 months. All VTAs were estimated in MNI space and left hemispheric VTAs were non-linearly warped to the right hemisphere.

First, we calculated an $\mathrm{N}$-image of the cohort by aggregating VTAs across the entire cohort. The $N$-image represents a heatmap and can be thresholded according to the number of times 
(n) each voxel in the map gets activated. To display the entire stimulation area of the cohort, we calculated the $\mathrm{N}$-image to illustrate all voxels, that were at least activated four times (representing $10 \%$ of small cohorts).

Second, we calculated a mean improvement map. To this end, each voxel was assigned the mean tremor improvement (part A) of all associated stimulation volumes activating this voxel. Each voxel had to be activated by at least four different stimulation volumes to be included in the analysis.

Third, we computed a significant improvement map adapted from Reich et al. ${ }^{26}$. In a first approach and closely akin to the method by Reich et al., the tremor improvement values associated with a given voxel were tested against the remaining improvement values not associated with this voxel by applying a two-sided t-test. With a significance level of 0.05 , we yielded a better improvement cluster as well as a worse improvement cluster. However, both clusters were relatively small and did not pass multiple testing correction with a false discovery rate of 0.05 (data not shown). The reasons for this probably comprise the non-normally distributed outcome data with a disproportionate number of excellent responders as well as the inhomogeneous stimulation map with a predominant number of voxels that get activated only a few times by different VTAs resembling a log-normal distribution. In a second approach, we shifted from a binary better-worse division to a division into suboptimal, good and excellent improvement clusters according to the $33 \%, 66 \%$ and $100 \%$ percentile. We intended to separate the suboptimal improvement cluster (corresponding to voxels with a $<50 \%$ tremor reduction) from a combined good and excellent improvement cluster (representing voxels with $>50 \%$ tremor reduction). Therefore, we applied a right-tailed Wilcoxon signed rank test. The null hypothesis was that the tremor improvement values associated with a given voxel were from a distribution with a median $m$. We chose $m$ to be the $33 \%$ percentile of all tremor improvement values. The alternate hypothesis was therefore that the associated tremor improvement values of a given voxel were from a distribution with a median greater than the $33 \%$ percentile. With a significance level of 0.05 , we identified two different clusters and multiple testing correction was applied with a false discovery rate of 0.05 . This resulted in a significant improvement map highlighting a statistically significant improvement cluster or statistically significant stimulation "sweet spot". The suboptimal improvement cluster did not pass multiple testing correction and was removed from further analysis.

The $\mathrm{N}$-image, mean improvement map together with their suboptimal and excellent responder clusters and significant improvement cluster were displayed in the three-dimensional DISTAL human brain atlas as well as superimposed onto the two-dimensional Schaltenbrand and Wahren stereotactic human brain atlas ${ }^{27}$. 


\section{Structural connectivity analysis}

The mean improvement map was displayed in relation to an average normative CTT based on high-quality diffusion imaging data of 32 healthy subjects from the Human Connectome Project. The method was recently described in detail in Dembek et al ${ }^{6}$. In summary, 62 CTTs were calculated using BEDPOSTX for estimating the local fiber orientation and PROBTRACKX2 for probabilistic tractography in $\mathrm{FSL}^{28}$. The contralateral dentate nucleus was chosen as seed region, while the contralateral superior cerebellar peduncle, the ipsilateral red nucleus, and the ipsilateral precentral gyrus served as waypoints. Each individual track frequency map was transformed into a track probability map into MNI space and probability values per voxel were averaged across all subjects.

\section{Outcome prediction model}

Finally, we validated the significant improvement map. To this end, we applied a stratified tenfold cross-validation, i.e., the data set of 213 stimulation volumes was randomly split into 90 percent training data (192 stimulation volumes) and 10 percent test data (21 stimulation volumes). The significant improvement map or sweet spot was computed with the training data. With the test data, we calculated the Euclidean distance from the centroid of a test stimulation volume to the centroid of the sweet spot. We also calculated the overlap volume between the test stimulation volume and the CTT. This was repeated ten times, i.e., each stimulation volume was a test volume once. Of note, the sweet spot was spatially stable across the stratified ten-fold cross-validation. A stratified five-fold cross-validation did not pass multiple testing correction to calculate the significant improvement map. Here, 80 percent of the data set, or 170 stimulation volumes, were not sufficient.

First, we intended to predict continuous tremor improvement. The factors "distance from VTA to the sweet spot" and the "probabilistic overlap volume between VTA and CTT" were fed as predictor variables into a support vector machine regression model. Tremor improvement was the response variable. Our assumption was that both proximity to the sweet spot and activation of the CTT would be meaningful predictors. Of note, a VTA may be close to the sweet spot but not activate the CTT and such a VTA would be assumed to result in less tremor improvement than a VTA with equal distance to the sweet spot but that activates the CTT. With respect to the model, we used a nonlinear regression model with a Gaussian Kernel function. Conceptually, this transforms the two predictors "distance" and "overlap volume" to a higherdimensional space. In that space, the algorithm then computes a linear function that is close to the response variable, while being as flat as possible (Matlab command fitrsvm). 
Second, we pursued another approach and intended to classify tremor improvement. Again the combination of factors distance and probabilistic overlap volume were used and fed into a classification ensemble (Matlab command fitcensemble with automatic hyperparameter optimization). The intention was to predict whether stimulation volumes would result in greater or less than $50 \%$ tremor improvement.

\section{Statistical analysis}

Data was analysed by applying descriptive non-parametric statistics after normality testing and visual inspection of the QQ-plots using SigmaPlot (Systat Software GmbH, San Jose, USA) and Matlab (The Mathworks, Natick, MA, USA) for baseline characteristics, tremor scores and outcome data. Nested linear mixed-effect models were used to test for the influence of different baseline covariates (sex, age, pre-operative tremor score, center, total electrical energy delivered as fixed factor and electrodes as random factor) on outcome (percentage change of Part A tremor subscores per hemisphere/contralateral hemibody). For statistical testing of the mean improvement map we applied a one-sided Wilcoxon signed rank test (see above) and multiple testing correction with false discovery rate of 0.05 . Data are presented as mean $\pm 95 \%$ confidence interval $(\mathrm{Cl})$ if not indicated otherwise. Statistical tests were two-tailed and a $\mathrm{p}$ value $<0.05$ was considered statistically significant. 


\section{Results}

\section{Clinical Data}

A total number of 119 patients from five European centers were included in the study. Table 1 shows the baseline demographic data for each center's patient cohort. In all patients, the Part A subscore was available after 12 months follow-up which corresponded to 237 hemispheres. The mean tremor reduction (Part A) per hemisphere across all centers was $64 \%$ (61 - $68 \%$, $95 \% \mathrm{Cl}$ ). Scatter plots of outcomes per center are shown in Figure 1. The results of the nested linear mixed effect model with age, gender, preoperative tremor intensity, total electrical energy delivered by DBS and center as fixed factors and patient and lead nested in patient as a random factors yielded only the two factors "preoperative tremor intensity" and "center" as significantly associated with outcome (Table 2). Individual tremor improvement varied substantially within the entire cohort (range $-25 \%$ to $100 \%$ tremor improvement). The outcome distribution of the entire cohort was right-shifted. The $33 \%$ percentile corresponded to a tremor improvement of $50 \%$ and the $67 \%$ percentile corresponding to an $80 \%$ tremor reduction.

The average (median and range) stimulation parameters of the cohort were $130 \mathrm{~Hz}(110-220$ $\mathrm{Hz}), 60 \mu \mathrm{s}(20-260 \mu \mathrm{s})$ and $2.6 \mathrm{~V}(0.9-7.4 \mathrm{~V})$.

\section{Stimulation Map}

Electrodes could be reconstructed in 107 patients (213 hemispheres). Twelve patients (16 hemispheres) had to be excluded from the image analysis as insufficient data quality of the postoperative CT or MRI scan did not allow a sufficiently precise detection of the electrode. Figure 2 displays the spatial distribution of the implanted leads in the Vim and PSA. The stimulation area covers large parts of the motor thalamus (beyond the Vim) and large parts of the PSA lateral to the RN, covering the $\mathrm{CZI}$ and extending into the entire posterior STN. As the results of the linear mixed effect model suggest the factor "center" to be significantly associated with outcome (with patients from the Bern cohort having a significantly worse outcome compared to the other centers), we compared the electrode implantation sites between centers. Based on both visual inspection and statistical testing, the electrodes from the Bern cohort are on average placed more medial compared to the other centers $(2.2 \pm 1.45 \mathrm{~mm}, \mathrm{p}<$ 0.001 ANOVA). For this reason, we decided not to correct for the factor "center" when constructing the stimulation map and outcome prediction models as we assume the difference between centers to result from different electrode placements.

The mean improvement map represents voxels that are associated with a certain clinical outcome. Displaying the whole map is visually not very informative, as areas associated with 
excellent tremor reduction are covered by areas of suboptimal tremor reduction, we thresholded the map according to our statistical approach by applying a partition into three clusters according to the $33 \%, 66 \%$ and $100 \%$ percentiles. We display the suboptimal responder cluster corresponding to a $<50 \%$ tremor reduction and an excellent responder cluster corresponding to a $>80 \%$ tremor reduction along with the surface volume of the entire unthresholded map in Figure 3. Whereas the area of excellent tremor reduction extends from the posterior aspect of the PSA to the central part of the Vim, the suboptimal responder voxels are located in the anterior and medial aspect of the PSA and extend to the Vop and the internal capsule laterally. The excellent responder cluster aligns visually well with the tractographybased CTT (Figure 4). Furthermore, we display the location of the identified significant good responder cluster that projects onto the margin of the posterior aspect of the PSA and Vim. The mean position of implanted DBS electrodes was $X=12.66, Y=-15.02, Z=-3.40$. In comparison, the centroid coordinates of the statistically significant good responder cluster were $X=13.63, Y=-15.13$ and $Z=-2.58 \mathrm{~mm}$ in MNI space. A projection of the stimulation map onto the stereotactic atlas of Schaltenbrand and Wahren is shown in Figure 5.

\section{Structural connectivity}

We displayed the suboptimal and excellent responder clusters as well as the significant good responder cluster in relation to the averaged normative CTT template (Figure 4). The CTT passed through the significant good responder cluster. This visual impression was confirmed by statistical testing. Voxels within the significant good responder cluster were more likely to contain the CTT as expressed by higher tract probability values compared to the rest of the map $(0.50 \pm 0.13$ versus $0.21 \pm 0.08 ; p<0.0001$, $t$-test $)$.

\section{Outcome prediction model}

Finally, we evaluated the predictive value of this map by applying a stratified ten-fold crossvalidation. The clinical outcome predicted from the significant improvement map by applying the support vector machine was associated with observed clinical outcome (mean $R^{2}=0.14$, $p=0.02$ ). Thus, the model estimations explained $14 \%$ of the variance of tremor score improvement. Using the distance to the sweet spot alone resulted in an $R^{2}$ of 0.06 , while using the probabilistic overlap volume alone yielded an $R^{2}$ of -0.17 (i.e., distance to sweet spot and probabilistic overlap volume alone would not be a good predictor). In comparison, an ordinary linear regression model with both distance and probabilistic overlap volume would have an $\mathrm{R}^{2}$ of 0.037 , underlining the better performance of the support vector machine. When applying a classification ensemble on the distance and overlap volume, we could correctly classify good 
and suboptimal responders (corresponding to $>50 \%$ and $<50 \%$ tremor reduction respectively) with a sensitivity of $89 \%$, a specificity of $57 \%$. The overall accuracy was $72 \%$. 


\section{Discussion}

To our knowledge, this is the largest multicentre study to integrate and compare clinical and neuroimaging data of patients with ET undergoing DBS. There was a considerable anatomical dispersion of implanted leads across included patients, which was reflected by an overall widespread area of stimulation covering large parts of the motor thalamus and the PSA. By applying voxel-wise statistical testing we could point out a "sweetspot" area of stimulation in the posterior part of the PSA and the inferior aspect of the Vim. The area of this "good responder cluster" coincided with the area of highest likelihood to contain the tractographybased normative CTT. Taken together, these findings suggest that stimulation of the posterior PSA and the Vim along the course of the CTT is associated with the highest likelihood of tremor suppression. The anatomical location of individual stimulation volumes was the overall best predictor of stimulation-induced tremor suppression with a sensitivity of $89 \%$ and a specificity of $57 \%$ to correctly identify responders with a greater than $50 \%$ tremor reduction based on a leave-one-out cross validation.

The five included centers placed their electrodes by slightly different targeting areas and approaches. Of note, the identified statistically significant improvement cluster (stimulation sweet spot) is closely related to the mean position of the implanted DBS electrodes (stimulation hotspot). The results can be interpreted in different ways. First, the exact location and extent of the identified significant improvement cluster is too restricted as it depends on the distribution of data and our chosen statistical approach by applying voxel-wise statistical testing and multiple testing correction. Areas of the map that contain more datapoints (which is the case at the stimulation hotspot) will more likely yield significant results and survive multiple testing correction. The fact that the stimulation area, which is associated with a good outcome, is more widespread than the identified significant stimulation sweet spot is in favor of this explanation. On the other hand, the identified significant improvement cluster could indeed reflect the "true" sweet spot and different the different centers just target around this optimal stimulation point based on years of clinical experience and targeting optimization. In line with this view, our findings are based on chronic stimulation settings that reflect empirical stimulation parameter adjustment to optimize the stimulation outcome.

\section{Anatomico-functional considerations}

The CTT projects from the deep cerebellar nuclei mainly to the contralateral thalamus ${ }^{29-31}$. The observation that several previous studies of DBS for ET reported satisfactory tremor reduction upon stimulation of slightly different thalamic and subthalamic targets including the PSA, cZI or prelemniscal radiations led to the formulation that the CTT might be the underlying neuroanatomical substrate stimulation of which would supress tremor ${ }^{3,5}, 7,32,33$. Since then, 
multiple studies from different groups studied the relationship between electrode locations, VTAs and the tractography-based CTT or functional connectivity patterns of good and poorresponders but came to different and sometimes contradictory conclusions. For instance, Akram et al. and Al-Fatly et al. identified a thalamic sweet-spot of optimal connectivity to the contralateral cerebellar dentate nucleus and the ipsilateral primary motor cortex (M1) whereas Middlebrooks identified a thalamic region that was connected to the supplementary motor area (SMA) and premotor area (PMA) to be correlated with better tremor reduction ${ }^{13,34,35}$. The results of the present work indicate that area of stimulation which corresponds to efficient tremor reduction coincides with a high probability to contain the CTT which is structurally connected to M1 and the contralateral dentate nucleus of the cerebellum - i.e., support the view of Akram's and Al-Fatly's work as well as the one by Dembek and co-workers ${ }^{6}$.

The present findings of the location of the probabilistic stimulation sweet spot at the transition between the ventral Vim and the PSA are in line with previous findings from an intraoperative electrophysiological study by Milosevic et $\mathrm{al}^{36}$. In their intraoperative study, the authors investigated the effects of high-frequency microstimulation on both neuronal firing and tremor suppression simultaneously by two closely placed microelectrodes. They found that $200 \mathrm{~Hz}$ high-frequency stimulation was significantly more effective to reduce tremor reduction and spontaneous cell firing in thalamic neurons compared to $100 \mathrm{~Hz}$ stimulation. Notably, they observed that the most ventroposterior stimulation sites had the best effect on tremor. The authors concluded that thalamic neuronal inhibition seems necessary for tremor reduction and may function in effect as a thalamic filter to uncouple thalamo-cortical from cortico-spinal reflex loops.

Although there is no question about the existence of cerebellothalamic connections from the deep cerebellar nuclei to the motor thalamus and their relevance in modulating movement, there is some controversy about which deep cerebellar nucleus is predominantly involved in tremor genesis. Based on anatomical tract tracer studies both the dentate nucleus as well as the interposed nuclei were shown to be connected to the motor thalamus and frontal regions including M1 and the supplementary motor area depending on the applied methodology and examined species ${ }^{30,31,37-39}$. The present study results suggest that high-frequency stimulation of cerebellothalamic fibers from the dentate nucleus to the Vim suppress tremor based on probabilistic tractography findings. However, we do not claim that fibers from the interposed nuclei also contribute to this effect. A definitive answer to this controversy can only be provided by well-designed post-mortem anatomical studies in humans

The presented results point towards a possible causal relationship of CTT-stimulation for tremor suppression. However, an involvement of the cZl for tremor genesis and stimulationinduced suppression cannot be ruled out. The $c Z$ Is bounded by the medial lemniscus 
posteriorly, the ascending cerebellothalamic fibres in the prelemniscal radiation anteriorly and the superior part of the STN laterally. As already pointed out by Plaha, the ZI provides a unique GABAergic link between the basal ganglia output nuclei and the cerebello-thalamo-cortical loop which places it in a key position to transmit synchronised oscillations generating tremor into these loops ${ }^{33}$. The close anatomical proximity of the CTT and the cZI makes it difficult, if not impossible to segregate DBS induced stimulation effects on these two structures with current neuroimaging and VTA-modelling approaches.

\section{Limitations}

Limitations of this study are the retrospective nature of the magnitude of used primary outcome data to calculate the probabilistic stimulation map and the two different outcome scales used across centers. However, both clinical tremor rating scales are validated and by using the percentage change of tremor, outcome data become normalized and reflect tremor improvement independent from the scale used. Second, the algorithm for image analysis including image normalization, lead reconstruction and electrode orientation is inherently prone to error. Although, most of the applied methods have been validated in phantom models before, without histological confirmation, DBS electrode reconstruction always remains presumptive. These inherent errors are very likely to limit the overall predictive value of the applied model. Third, we used a VTA model that assumes an isotropic environment, though the leads had been implanted in a region with moderate anisotropy. Diffusion weighted imaging and an expansion of the VTA model would be necessary to provide better patient-specific fiber tracking and to accommodate anisotropy. Moreover, each algorithm dedicated to coregistration and normalization involves inherent errors and impact the overall calculation precision. Fourth, we used normative connectome data to estimate the average CTT. Although these normative connectome atlases do not represent patient-specific connectivity, they in turn have the benefit of high signal-to-noise ratios. Furthermore, the results of a recent study by Wang et al. put into perspective the limitations of normative versus patient-specific connectivity analysis ${ }^{40}$. In addition, we did not integrate side-effects into our probabilistic modelling approach. One major reason is the retrospective design of the study and missing data. However, it was regular clinical practice across all centers to titrate and optimize the stimulation parameters by balancing best possible tremor suppression while avoiding limiting stimulationinduced side-effects. It is therefore reasonable to assume that the probabilistic stimulation map in a certain way contains indirect information about side-effects. Nevertheless, future research needs to focus on objectively and quantitatively assessed side-effects.

Lastly, we tested different outcome-prediction models based on different assumptions and variables to get to the final results that were not controlled or corrected for these multiple 
approaches. Still, the optimized algorithm underlying the outcome-prediction model can only explain $14 \%$ of the variance of the observed outcome if we seek to predict individual percentage tremor reduction. We suspect that the overall widespread stimulation area and the noisy nature of both clinical outcome and imaging data are the main reason that our applied models fail to predict individual continuous tremor improvement more accurately. Because of the low R-squared values, we further tested a classification with a random forest to predict a binarized outcome instead. The results of the cross-validation and their predictive value need to be interpreted with caution and the presented results are mainly descriptive until confirmed in independent datasets, ideally through prospective testing. 


\section{Author contributions}

AN and TD, AH, AK, TZA, TN and CP contributed to the conception and design of the study; AN, SB, $B A F, T D, M B, A G, D K, L L, I D, A S A, V V V, T Z A, R S, T N$ and EF contributed to the acquisition and analysis of data; AN, SB, AH, LL, ID, VVV, TD, TZA, AG, MB and CP contributed to drafting the text or preparing the figures.

\section{Potential Conflicts of interest}

Nothing to report.

\section{Data availability}

The entire stimulation map as well as the significant improvement cluster will be made publically available as a NIfTI files. 


\section{References}

1. Lozano AM. Vim thalamic stimulation for tremor. Archives of medical research. 2000;31:266-269.

2. Benabid AL, Pollak P, Gervason C, Hoffmann D, Gao DM, Hommel M, et al. Long-term suppression of tremor by chronic stimulation of the ventral intermediate thalamic nucleus. Lancet. 1991;337:403-406.

3. Carrillo-Ruiz JD, Velasco F, Jimenez F, Castro G, Velasco AL, Hernandez JA, et al. Bilateral electrical stimulation of prelemniscal radiations in the treatment of advanced Parkinson's disease. Neurosurgery. 2008;62:347-357; discussion 357-349.

4. Kitagawa M, Murata J, Kikuchi S, Sawamura Y, Saito H, Sasaki H, et al. Deep brain stimulation of subthalamic area for severe proximal tremor. Neurology. 2000;55:114-116.

5. Coenen VA, Allert N, Paus S, Kronenburger M, Urbach H, Madler B. Modulation of the cerebello-thalamo-cortical network in thalamic deep brain stimulation for tremor: a diffusion tensor imaging study. Neurosurgery. 2014;75:657-669; discussion 669-670.

6. Dembek TA, Petry-Schmelzer JN, Reker P, Wirths J, Hamacher S, Steffen J, et al. PSA and VIM DBS efficiency in essential tremor depends on distance to the dentatorubrothalamic tract. Neurolmage Clinical. 2020;26:102235.

7. Fiechter M, Nowacki A, Oertel MF, Fichtner J, Debove I, Lachenmayer ML, et al. Deep Brain Stimulation for Tremor: Is There a Common Structure? Stereotactic and functional neurosurgery. 2017;95:243-250.

8. Cury RG, Fraix V, Castrioto A, Perez Fernandez MA, Krack P, Chabardes S, et al. Thalamic deep brain stimulation for tremor in Parkinson disease, essential tremor, and dystonia. Neurology. 2017;89:1416-1423.

9. Sandoe C, Krishna V, Basha D, Sammartino F, Tatsch J, Picillo M, et al. Predictors of deep brain stimulation outcome in tremor patients. Brain stimulation. 2018;11:592-599.

10. Papavassiliou E, Rau G, Heath S, Abosch A, Barbaro NM, Larson PS, et al. Thalamic deep brain stimulation for essential tremor: relation of lead location to outcome. Neurosurgery. 2004;54:1120-1129; discussion 1129-1130.

11. Bhatia KP, Bain P, Bajaj N, Elble RJ, Hallett M, Louis ED, et al. Consensus Statement on the classification of tremors. from the task force on tremor of the International Parkinson and Movement Disorder Society. Movement disorders : official journal of the Movement Disorder Society. 2018;33:75-87.

12. Barbe MT, Reker P, Hamacher S, Franklin J, Kraus D, Dembek TA, et al. DBS of the PSA and the VIM in essential tremor: A randomized, double-blind, crossover trial. Neurology. 2018;91:e543-e550.

13. Al-Fatly B, Ewert S, Kubler D, Kroneberg D, Horn A, Kuhn AA. Connectivity profile of thalamic deep brain stimulation to effectively treat essential tremor. Brain : a journal of neurology. 2019;142:3086-3098.

14. Bot M, van Rootselaar F, Contarino MF, Odekerken V, Dijk J, de Bie R, et al. Deep Brain Stimulation for Essential Tremor: Aligning Thalamic and Posterior Subthalamic Targets in 1 Surgical Trajectory. Operative neurosurgery. 2018;15:144-152.

15. Nowacki A, Bogdanovic M, Sarangmat N, Fitzgerald J, Green A, Aziz TZ. Revisiting the rules for anatomical targeting of ventralis intermediate nucleus. Journal of clinical neuroscience : official journal of the Neurosurgical Society of Australasia. 2019;68:97-100.

16. Nowacki A, Debove I, Rossi F, Schlaeppi JA, Petermann K, Wiest R, et al. Targeting the posterior subthalamic area for essential tremor: proposal for MRI-based anatomical landmarks. Journal of neurosurgery. 2018;131:820-827.

17. Bain PG, Findley LJ, Atchison P, Behari M, Vidailhet M, Gresty M, et al. Assessing tremor severity. Journal of neurology, neurosurgery, and psychiatry. 1993;56:868-873. 
18. Stacy MA, Elble RJ, Ondo WG, Wu SC, Hulihan J, group TRSs. Assessment of interrater and intrarater reliability of the Fahn-Tolosa-Marin Tremor Rating Scale in essential tremor. Movement disorders : official journal of the Movement Disorder Society. 2007;22:833838.

19. Horn A, Li N, Dembek TA, Kappel A, Boulay C, Ewert S, et al. Lead-DBS v2: Towards a comprehensive pipeline for deep brain stimulation imaging. Neurolmage. 2019;184:293-316. 20. Avants BB, Epstein CL, Grossman M, Gee JC. Symmetric diffeomorphic image registration with cross-correlation: evaluating automated labeling of elderly and neurodegenerative brain. Medical image analysis. 2008;12:26-41.

21. Ewert S, Plettig P, Li N, Chakravarty MM, Collins DL, Herrington TM, et al. Toward defining deep brain stimulation targets in MNI space: A subcortical atlas based on multimodal MRI, histology and structural connectivity. Neurolmage. 2018;170:271-282.

22. Husch A, M VP, Gemmar P, Goncalves J, Hertel F. PaCER - A fully automated method for electrode trajectory and contact reconstruction in deep brain stimulation. Neurolmage Clinical. 2018;17:80-89.

23. Dembek TA, Hoevels M, Hellerbach A, Horn A, Petry-Schmelzer JN, Borggrefe J, et al. Directional DBS leads show large deviations from their intended implantation orientation. Parkinsonism \& related disorders. 2019;67:117-121.

24. Hellerbach A, Dembek TA, Hoevels M, Holz JA, Gierich A, Luyken K, et al. DiODe: Directional Orientation Detection of Segmented Deep Brain Stimulation Leads: A Sequential Algorithm Based on CT Imaging. Stereotactic and functional neurosurgery. 2018;96:335-341. 25. Oostenveld R, Fries P, Maris E, Schoffelen JM. FieldTrip: Open source software for advanced analysis of MEG, EEG, and invasive electrophysiological data. Computational intelligence and neuroscience. 2011;2011:156869.

26. Reich MM, Horn A, Lange F, Roothans J, Paschen S, Runge J, et al. Probabilistic mapping of the antidystonic effect of pallidal neurostimulation: a multicentre imaging study. Brain : a journal of neurology. 2019;142:1386-1398.

27. Schaltenbrand G, Wahren W. Atlas For Stereotaxy of the Human Brain, 2nd Edition: Thieme, Stuttgart, 1977.

28. Behrens TE, Berg HJ, Jbabdi S, Rushworth MF, Woolrich MW. Probabilistic diffusion tractography with multiple fibre orientations: What can we gain? Neurolmage. 2007;34:144155.

29. Gallay MN, Jeanmonod D, Liu J, Morel A. Human pallidothalamic and cerebellothalamic tracts: anatomical basis for functional stereotactic neurosurgery. Brain structure \& function. 2008;212:443-463.

30. Hoover JE, Strick PL. The organization of cerebellar and basal ganglia outputs to primary motor cortex as revealed by retrograde transneuronal transport of herpes simplex virus type 1. J Neurosci. 1999;19:1446-1463.

31. Orioli PJ, Strick PL. Cerebellar connections with the motor cortex and the arcuate premotor area: an analysis employing retrograde transneuronal transport of WGA-HRP. The Journal of comparative neurology. 1989;288:612-626.

32. Blomstedt P, Sandvik U, Linder J, Fredricks A, Forsgren L, Hariz MI. Deep brain stimulation of the subthalamic nucleus versus the zona incerta in the treatment of essential tremor. Acta neurochirurgica. 2011;153:2329-2335.

33. Plaha P, Khan S, Gill SS. Bilateral stimulation of the caudal zona incerta nucleus for tremor control. Journal of neurology, neurosurgery, and psychiatry. 2008;79:504-513.

34. Akram H, Dayal V, Mahlknecht P, Georgiev D, Hyam J, Foltynie T, et al. Connectivity derived thalamic segmentation in deep brain stimulation for tremor. Neurolmage Clinical. 2018;18:130-142.

35. Middlebrooks EH, Tuna IS, Almeida L, Grewal SS, Wong J, Heckman MG, et al. Structural connectivity-based segmentation of the thalamus and prediction of tremor improvement following thalamic deep brain stimulation of the ventral intermediate nucleus. Neurolmage Clinical. 2018;20:1266-1273.

36. Milosevic L, Kalia SK, Hodaie M, Lozano AM, Popovic MR, Hutchison WD. Physiological mechanisms of thalamic ventral intermediate nucleus stimulation for tremor suppression. Brain : a journal of neurology. 2018;141:2142-2155. 
37. Aumann TD, Rawson JA, Finkelstein DI, Horne MK. Projections from the lateral and interposed cerebellar nuclei to the thalamus of the rat: a light and electron microscopic study using single and double anterograde labelling. The Journal of comparative neurology. 1994;349:165-181.

38. Jorntell $\mathrm{H}$, Ekerot CF. Topographical organization of projections to cat motor cortex from nucleus interpositus anterior and forelimb skin. J Physiol. 1999;514 ( Pt 2):551-566.

39. Rouiller EM, Liang F, Babalian A, Moret V, Wiesendanger M. Cerebellothalamocortical and pallidothalamocortical projections to the primary and supplementary motor cortical areas: a multiple tracing study in macaque monkeys. The Journal of comparative neurology. 1994;345:185-213.

40. Wang Q, Akram H, Muthuraman M, Gonzalez-Escamilla G, Sheth SA, Oxenford S, et al. Normative vs. patient-specific brain connectivity in deep brain stimulation. Neurolmage. 2021;224:117307. 


\section{Figure Legends}

Figure 1: Scatterplot of percentage tremor reduction per center and outcome frequency distribution. 12 months following surgery the mean tremor reduction based on the part $A$ clinical tremor rating scale subscore was significantly lower in the Bern cohort (48 $\pm 29 \%)$ compared to the other participating centers (ranging between 63 and $69 \%$ tremor reduction) (A). The histogam of postoperative tremor improvement (Part A subscale) for all centers reveals a left-skewed distribution (B).

Figure 2: Electrode locations and overall stimulation area ( $\mathrm{N}$-image) Pseudo threedimensional representations of all reconstructed implanted DBS leads of the multicentre cohort from a posterior view $(A)$ and view from top $(B)$. (The $N_{1}$-image represents the visualization of all voxels activated at least once from the entire cohort, lateralized on the right hemisphere is shown in a view from top top (C), from medial (D) and from posterior (E) and represents the entire area of stimulation across all patients.

Figure 3: Mean clinical improvement map, "excellent" and "suboptimal" responder cluster. Pseudo-three-dimensional views of the mean clinical improvement map is shown in a view from top $(A)$, medial $(B)$ and $(C)$ posterior. The map is color-coded from yellow (low) to red (high) and represents the mean tremor reduction values per voxel (see color bar). For better visualization of the sub-zones of the map it is further thresholded according to the 67$100^{\text {th }}$ percentile to display an "excellent responder cluster" in a view from top (D), medial (E) and $(F)$ posterior as well as according to the $0-33^{\text {rd }}$ percentile to display "suboptimal" responder voxels a view from top $(\mathrm{G})$, medial $(\mathrm{H})$ and $(\mathrm{I})$ posterior. Voxels that were located anterior and medial in the PSA as well as lateral with close relation to the internal capsule were associated with a lower tremor reduction whereas voxels in an (ii) intermediate area of the posterior PSA extending through the vertical axis in the central aspect of the Vim were associated with high tremor reduction.

Figure 4: Anatomical relation of the CTT to the stimulation map. Pseudo-threedimensional view from posterior to display the anatomical relationship of the tractographybased cerebello-thalamic-tract (CTT, blue) to the "excellent responder cluster" (A, red), "suboptimal responder cluster" (B, yellow) and "significant good responder cluster" ( $C$, dark red). Whereas voxels associated with $>80 \%$ tremor reduction values are clustered along the 
CTT, voxels with $<50 \%$ tremor reduction are predominantly more distant to the CTT. The significant good responder cluster co-localizes with the center of the CTT. The anatomical relation of the CTT outlined in blue, the Vim, STN and RN outlined in black as well as the significant good responder cluster outlined in white are shown in an axial (D), coronal (E) and sagittal $(F)$ reconstruction. The level of the axial, sagittal and coronal sections of the 2D reconstructions of the whole map is indicated in the left upper inset and indicated by the corresponding MNI coordinates.

Figure 5: Clinical mean improvement image projected onto the Schaltenbrand and Wahren Stereotactic Atlas of the Human Brain. The clinical mean improvement image derived in MNI space is projected onto the Schaltenbrand and Wahren atlas at corresponding axial $(A, B)$, sagittal $(C)$ and coronal planes $(D)$. The MNI- and their corresponding AC-PCcoordinates are indicated in the lower left and lower right part of each panel. Areas with associated good to excellent tremor reduction predominantly cover the the prelemniscal radiations (Ra.prl) and the inferior aspect of the Vim (V.im.e). a smaller cluster is located in the dorsolateral part of the STN. Areas associated with suboptimal tremor control cover the medial aspect of the PSA close to the red nucleus $(\mathrm{Ru})$ and the internal capsule. 


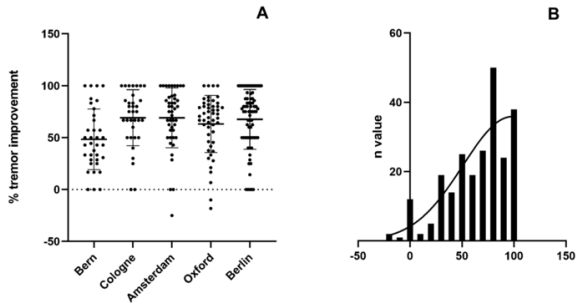

ANA_26324_Figure 1.tif 

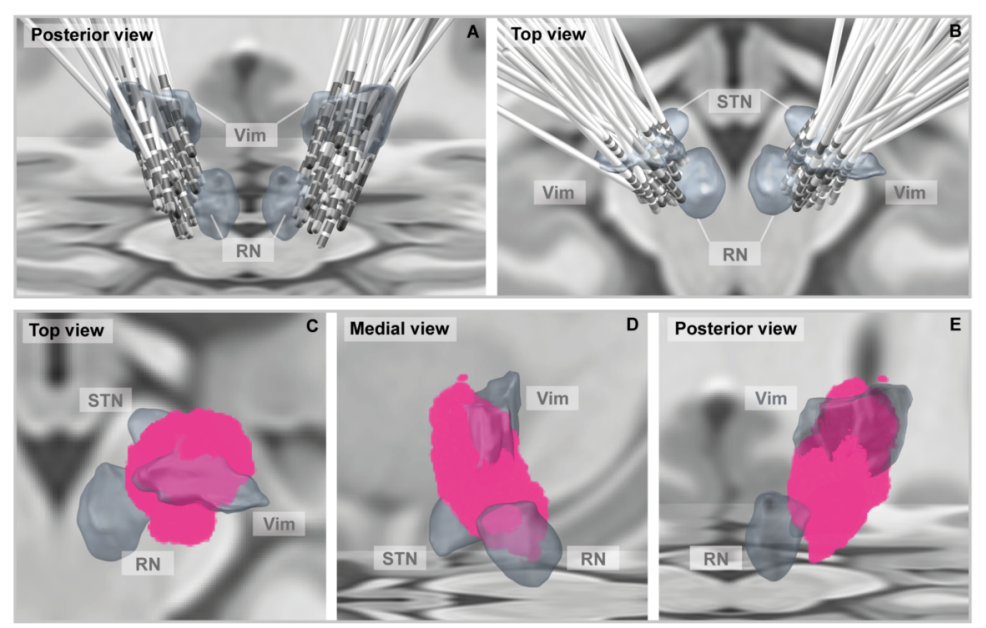

ANA_26324_Figure 2.tif 


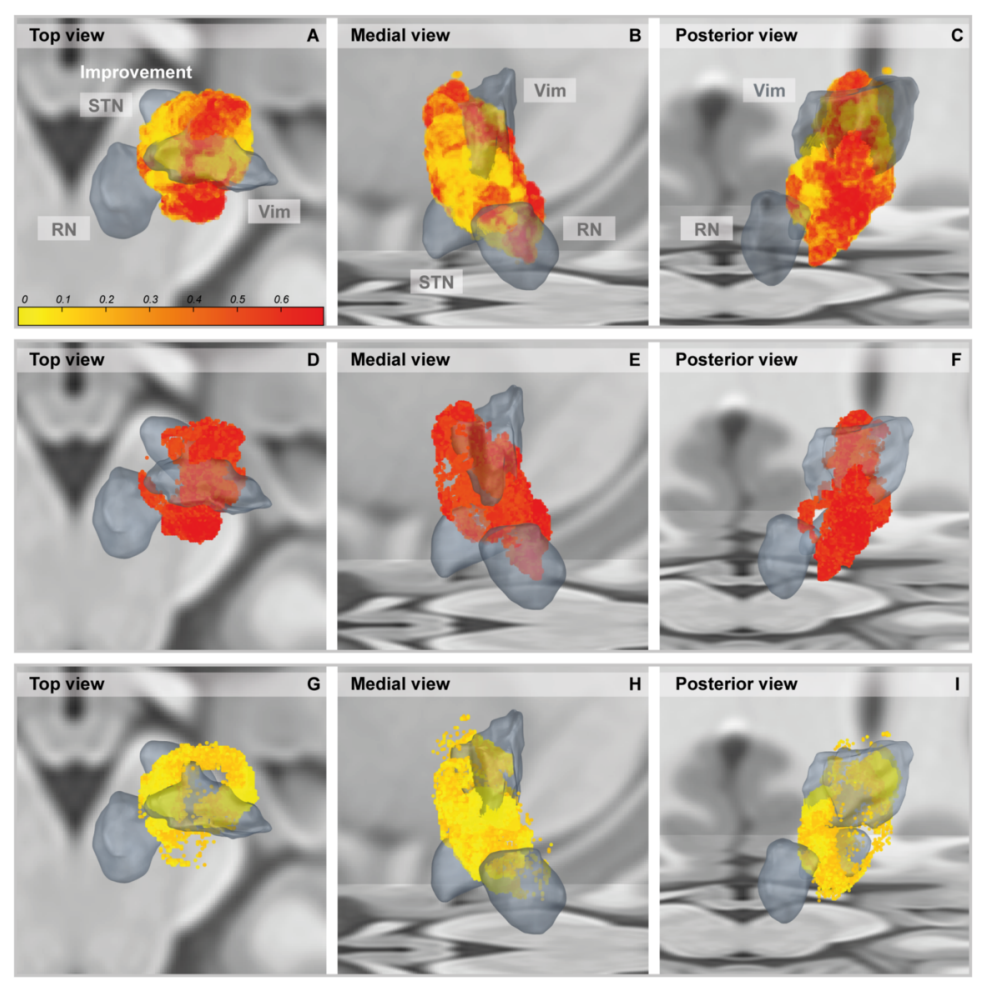

ANA_26324_Figure 3.tif 


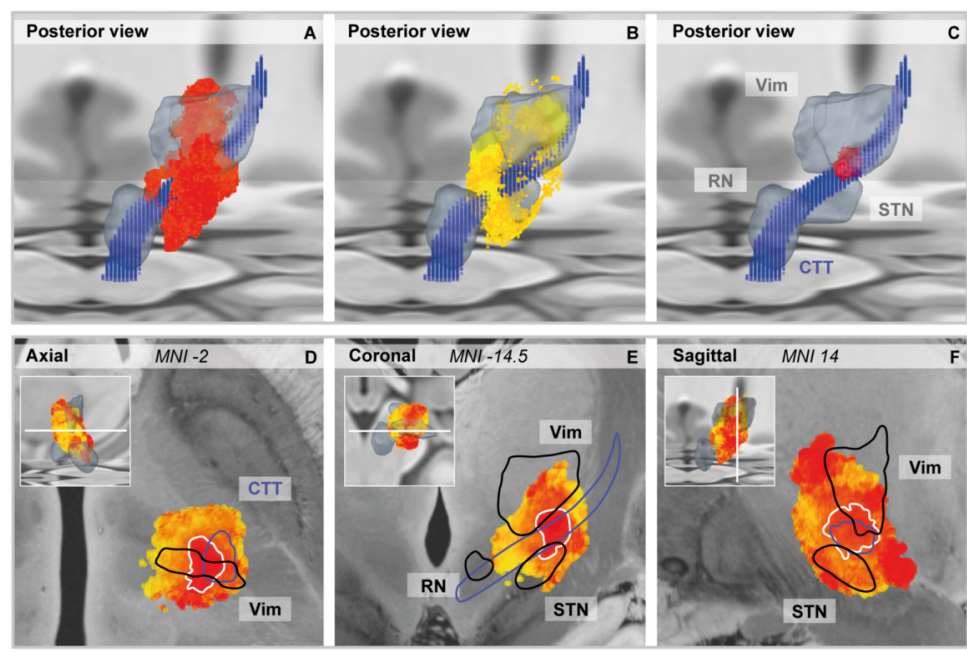

ANA_26324_Figure 4.tif 


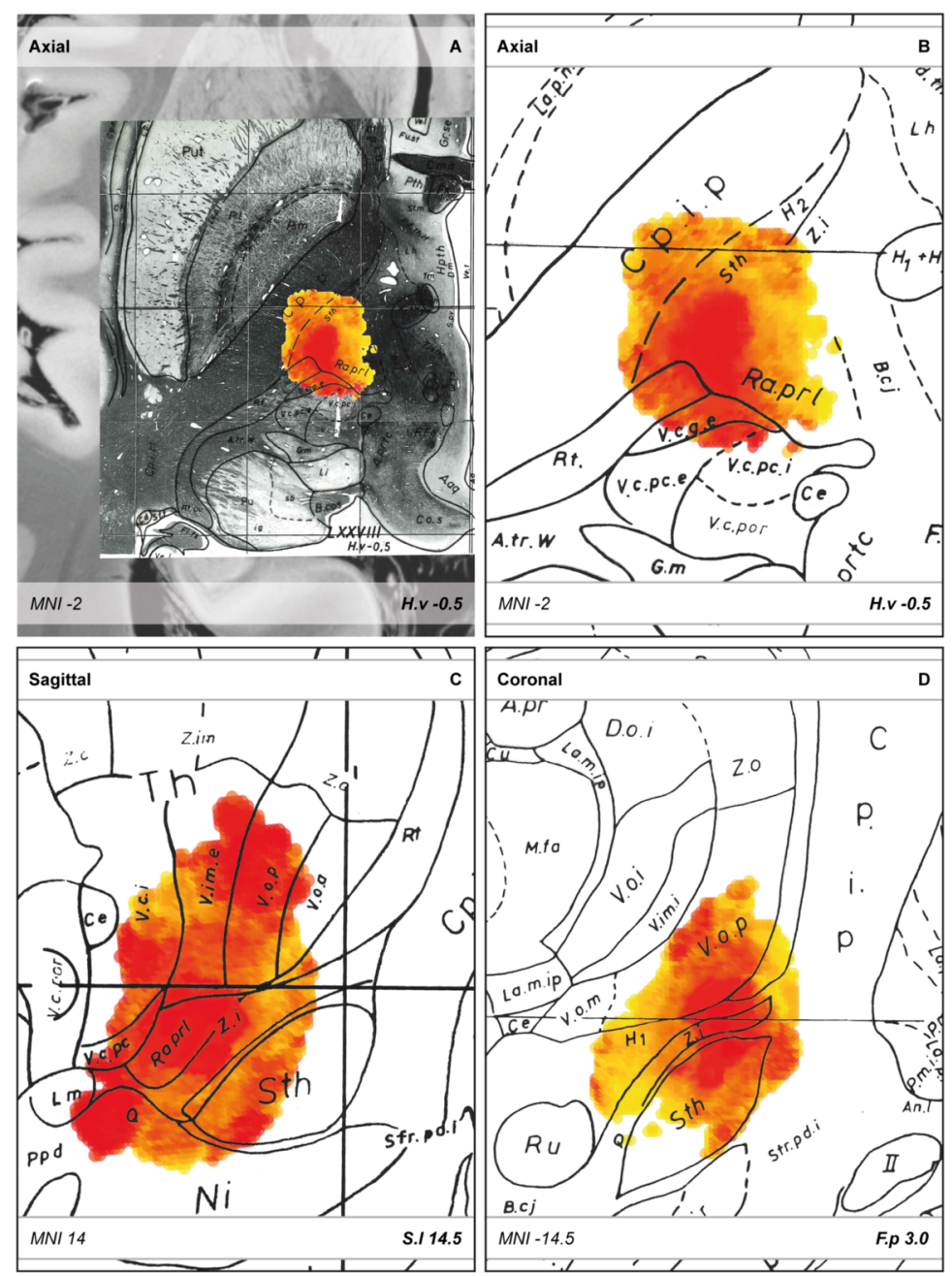

ANA_26324_Figure 5.tif 